\title{
Tourniquet use in total knee arthroplasty: a meta-analysis
}

\author{
Ta-Wei Tai $\cdot$ Chii-Jeng Lin $\cdot$ I-Ming Jou $\cdot$ \\ Chih-Wei Chang $\cdot$ Kuo-An Lai $\cdot$ Chyun-Yu Yang
}

Received: 21 April 2010/Accepted: 15 November 2010/Published online: 15 December 2010

(C) The Author(s) 2010. This article is published with open access at Springerlink.com

\begin{abstract}
Purpose The use of an intraoperative tourniquet for total knee arthroplasty (TKA) is a common practice. However, the effectiveness and safety are still questionable. A systematic review was conducted to examine that whether using a tourniquet in TKA was effective without increasing the risk of complications.

Methods A comprehensive literature search was done in PubMed Medicine, Embase, and other internet database. The review work and the following meta-analysis were processed to evaluate the role of tourniquet in TKA.

Results Eight randomized controlled trials and three high-quality prospective studies involving 634 knees and comparing TKA with and without the use of a tourniquet were included in this analysis. The results demonstrated that using a tourniquet could decrease the measured blood loss but could not decrease the calculated blood loss, which indicated actual blood loss. Patients managed with a tourniquet might have higher risks of thromboembolic complications. Using the tourniquet with late release after wound closure could shorten the operation time; whereas early release did not show this benefit.

Conclusions The current evidence suggested that using tourniquet in TKA may save time but may not reduce the blood loss. Due to the higher risks of thromboembolic
\end{abstract}

T.-W. Tai · C.-J. Lin · I.-M. Jou · C.-W. Chang · K.-A. Lai · C.-Y. Yang $(\square)$

Department of Orthopaedics, National Cheng Kung University Hospital, 138 Sheng-Li Rd., Tainan, Taiwan

e-mail: cyyang@mail.ncku.edu.tw

T.-W. Tai

Department of Orthopaedics, Buddhist Dalin Tzu Chi General Hospital, Chiayi, Taiwan complications, we should use a tourniquet in TKA with caution.

Keywords Tourniquet $\cdot$ Knee arthroplasty $\cdot$ Blood loss · Thromboembolism · Meta-analysis

\section{Introduction}

Total knee arthroplasty (TKA) is associated with significant blood loss, for which blood transfusion might be necessary. The role of the tourniquet is controversial, though it is widely used by orthopedic surgeons. Its use was believed to be effective in decreasing intraoperative blood loss and creating a bloodless surgical field, which theoretically would facilitate the cementing technique and other surgical procedures. However, reactive blood flow reached its peak within $5 \mathrm{~min}$ after the tourniquet had been released [16]. The tourniquet controls intraoperative blood loss, but cannot stop postoperative blood loss. The role of the tourniquet in the total blood loss of patients undergoing TKA is still questionable.

In addition, the benefits of the tourniquet must be balanced against the shortages and risks. Patients who were managed with a tourniquet during the operation often complained of thigh pain. This was possibly caused by the direct pressure of an inflated tourniquet on the nerves and local soft tissues. Limb swelling and increased soft tissue tension caused by reactive hyperperfusion after tourniquet deflation may also contribute to the wound pain.

Thromboembolism is one of the most serious postoperative complications in orthopedic surgery. Clinically, the use of the pneumatic tourniquet is considered to be one of the most important risk factors for symptomatic thromboembolism $[13,37]$. The incidence of deep vein thrombosis 
(DVT) after TKA performed with a tourniquet was reported to be up to $72-84 \%$ in earlier studies [20, 31], but this has been reduced with the advances in thromboembolic prophylaxis. The deflation of a tourniquet also changes the hemodynamic status [26] and allows a substantial amount of emboli to travel from the lower limb into the right atrium, right ventricle, and pulmonary artery [3]. This phenomenon may be predictive of further clinical pulmonary embolic events.

Other tourniquet-related complications have been reported in the literature and include nerve palsy [10], vascular injuries [15], rhabdomyolysis [24], and subcutaneous thigh fat necrosis [32]. The application of a tourniquet also tethers the quadriceps mechanism and thus alters the intraoperative patellofemoral tracking [11, 14, 19]. This change may affect the surgeon's judgment on soft tissue balancing and the decision to perform lateral release.

Several randomized controlled trials regarding the use of tourniquets have been published, but no consensus has been reached. Heterogeneous data had been reported in these trials. Thus, a meta-analysis was conducted to assess the effects of using a tourniquet for primary TKA, with the aim of quantifying the effect of the tourniquet on reducing blood loss and assessing the possible risks associated with tourniquets.

\section{Methods}

We followed the methodological guidelines outlined by the Cochrane Collaboration (Oxford, United Kingdom) [9] in conducting this meta-analysis. The findings were reported according to the recommendations outlined in the Quality of Reporting of Meta-Analyses (QUOROM) statement [21].

\section{Literature search}

Two of the authors completed a search of the electronic databases, including the Cochrane Central Register of Controlled Trials (2009), PubMed Medline (1966-2009), and Embase (1980-2009). We used the following search terms and Boolean operators: "tourniquet" AND ("knee arthroplasty" OR "knee replacement"). Editorials and commentaries from major orthopedic journals were also considered for further information on trials of interest. We also used the internet search engine "Google", searching under the keyword "tourniquet" combined with "knee," "arthroplasty," “joint replacement," "blood loss," “deep vein thrombosis," or "pulmonary embolism." The reference lists of the relevant articles were also looked through for any further relevant studies.
Article selection and validity assessment

We included prospective trials comparing patients undergoing TKA with a tourniquet or not. Retrospective studies or case series were excluded. The blood loss and incidence of thromboembolic events were considered as primary outcomes. After reviewing the titles and abstracts of the studies, we determined whether the study was appropriate for retrieval. We then appraised the studies and identified those eligible for inclusion in our analysis. Using the Consolidated Standards of Reporting Trials (CONSORT) checklist of 22 items [22], we assessed the quality of reporting in each article. All disagreements were resolved by consensus.

\section{Data extraction}

Two authors independently extracted the data from the articles. Whenever necessary, we contacted the authors of the studies for the missing data or further information. Encountering any disagreement, we reached a consensus by discussion within the review team. The following data were extracted: (1) participant demographics; (2) indication for TKA; (3) operative methods and usage of the tourniquet; (4) blood loss measures, including the intraoperative blood loss, amount of drainage, total measured blood loss, and calculated blood loss; (5) operation time; (6) thromboembolic events and other tourniquet-related complications. We also contacted the authors for missing data.

\section{Statistical analysis}

The outcomes were collected using variance-weighted means. We tested for the presence of heterogeneity with the use of a standard chi-square test (with a level of significance of $P=0.1$ ) and the $I^{2}$ statistic [8]. An $I^{2}$ statistic value of $>50 \%$ was considered to indicate substantial heterogeneity. Random-effects analysis [4] was used for comparing trials showing heterogeneity while fixed-effects analysis [17] was used for comparing trials without showing heterogeneity. We calculated the mean difference or relative risk for all outcomes. The meta-analysis was carried out using the RevMan 5 software package (Cochrane Collaboration, Oxford, United Kingdom).

\section{Results}

Identified trials

A total of 810 articles were retrieved in the search. Eight randomized controlled trials and three high-quality 
prospective studies (evidence-based level II) comparing TKA with and without the use of a tourniquet were identified and included in the meta-analysis (Table 1). These studies involved a total of 634 TKAs. The characteristics of operation methods were summarized in Table 2. They included some co-factors that might influence the blood loss in TKA.

\section{Methodology}

The methodological quality of the included studies is summarized in Table 1. The inclusion criteria were reported in all studies, but the exclusion criteria were made known in only four studies. No study reported the method of random sequence generation and the detailed implementation of randomization. Baseline characteristics were not reported in one study [37], which could potentially bias the results and limit the generalizability of the findings.

\section{Outcome analysis}

Intraoperative blood loss and postoperative blood drainage were reported in five studies. The pooling data showed that using a tourniquet significantly decreased the blood loss during the operation but slightly increased the postoperative blood loss (Fig. 1c, d). In terms of total measured blood loss (the sum of intraoperative and postoperative blood loss), the pooling data of the five studies favored using a tourniquet (Fig. 1a). However, after taking out an extreme data [7], the pooling result of the remaining studies showed no significant difference between these two groups.

Three studies mentioned calculated blood loss, which involved taking the patients' blood volume and change in hematocrit levels into consideration, and was thought to be an exact representation of the true blood loss. The pooling results surprisingly revealed slightly less calculated blood loss in the non-tourniquet group (Fig. 1b).

With respect to thromboembolic events after surgery, eight studies provided data on the incidence of sonographic or clinical DVT and pulmonary embolism. The pooling results found that the risk of clinical thromboembolic events was significantly higher in the tourniquet group than in the non-tourniquet group (Fig. 2a). Symptomatic thromboembolic events occurred in 13.0 and $6.1 \%$ of patients in the tourniquet and non-tourniquet group, respectively (Table 3 ). In the subgroup analysis, both symptomatic DVT and pulmonary embolism occurred more often in the tourniquet group than in the non-tourniquet group, although there was no statistical difference (Fig. 2b, c). One study [36] claimed there were no DVTs in either group. Two studies investigated the incidence of DVT using sonographic image examinations $[5,36]$. The diversity of results indicated that the sonographic examination was operator dependent. The pooled results showed no difference between the tourniquet and non-tourniquet groups (Fig. 2d).

Transesophageal echocardiography was used to detect intraoperative emboli in two studies [12,37], and both of them reported that snowstorm-like echogenic particles were observed after deflation of the tourniquet in all

Table 1 Characteristics of the studies

\begin{tabular}{|c|c|c|c|c|c|}
\hline & $\begin{array}{l}\text { No. in } \\
\text { tourniquet } \\
\text { group }\end{array}$ & $\begin{array}{l}\text { No. in } \\
\text { non-tourniquet } \\
\text { group }\end{array}$ & $\begin{array}{l}\text { Mean age } \\
\text { (tourniquet/ } \\
\text { non-tourniquet) }\end{array}$ & Type of cohort & $\begin{array}{l}\text { CONSORT } \\
\text { methodology } \\
\text { score }\end{array}$ \\
\hline Abdel-Salam [1] & 40 & 40 & $67.2 / 65.4$ & Randomized controlled trial & 9 \\
\hline Harvey [7] & 36 & 28 & $63.2 / 61.4$ & $\begin{array}{l}\text { Part of a prospective double-blinded } \\
\text { study on thromboprophylaxis }\end{array}$ & NA \\
\hline Wakankar [36] & 37 & 40 & $72.5 / 71.8$ & Randomized controlled trial & 9 \\
\hline Aglietti [2] & 10 & 10 & $70 / 68$ & Randomized controlled trial & 12 \\
\hline Tetro [33] & 33 & 30 & $69.8 / 69.8$ & Randomized controlled trial & 15 \\
\hline Kato [12] & 22 & 24 & $63 / 65$ & Randomized controlled trial & 9 \\
\hline Vandenbussche [35] & 40 & 40 & $80.3 / 72.5$ & Randomized controlled trial & 11 \\
\hline Wauke [37] & 19 & 18 & $68 / 69$ & Randomized controlled trial & 9 \\
\hline Nishiguchi [23] & 14 & 25 & $71.2 / 73.1$ & High-quality prospective study & NA \\
\hline Fukuda [5] & 27 & 21 & $72 / 74$ & High-quality prospective study & NA \\
\hline Li [18] & 40 & 40 & $71 / 70$ & Randomized controlled trial & 11 \\
\hline Total & 318 & 316 & - & - & - \\
\hline
\end{tabular}

NA not applicable 


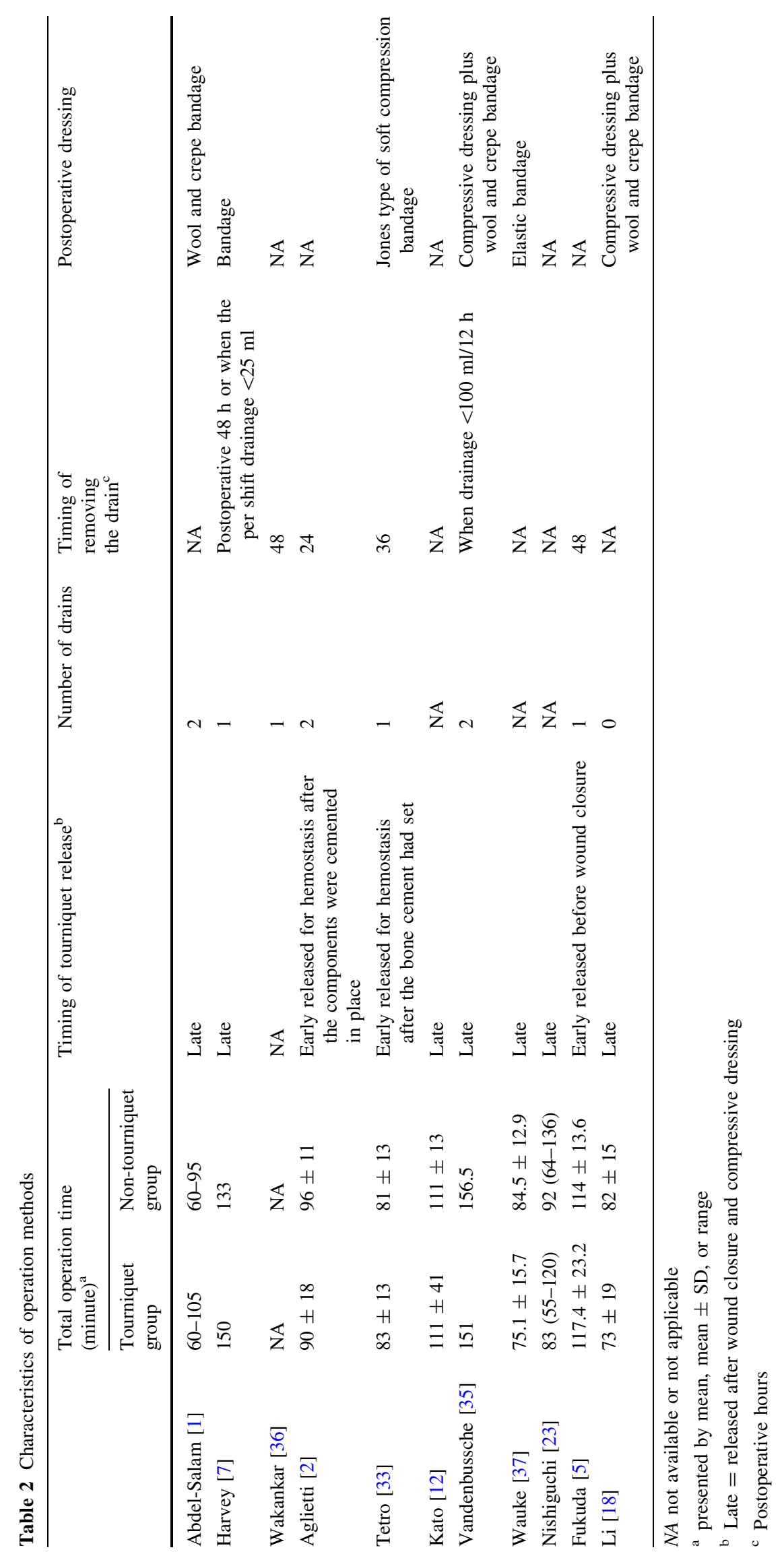


Fig. 1 a Total measured blood loss: The pooling data of the five studies favored using a tourniquet. However, Harvey et al. [7] had an extreme result. The total measured blood loss in the non-tourniquet group was twice that in the tourniquet group (1,493 versus $709 \mathrm{ml})$ probably because of cementless technique and prolonged surgical time. After taking out this result, the pooling data of the remaining studies showed no significant difference. b Calculated blood loss: The pooling results revealed slightly less calculated blood loss in the non-tourniquet group.

c Intraoperative blood loss: All of five studies showed that using a tourniquet significantly decreased blood loss during the operation. The mean

intraoperative blood loss in operations without a tourniquet ranged from 295 to $631 \mathrm{ml}$. d Postoperative blood loss: The pooling data revealed a slight decrease in the drained volume of the non-tourniquet group.

The means of the total drained blood ranged from 290 to $528.5 \mathrm{ml}$ in the tourniquet group and 145 to $661.6 \mathrm{ml}$ in the nontourniquet group. Overall, pooling date showed that using a tourniquet in TKA could reduce the intraoperative blood loss and total measured blood loss, but could not decrease the calculated blood loss a Total measured Blood Loss

Std. Mean Difference

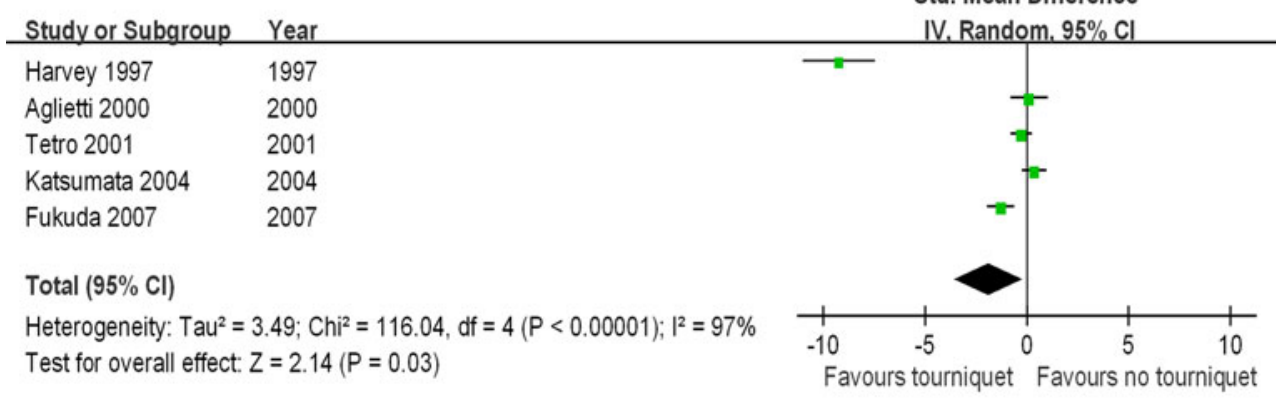

b Calculated Blood Loss

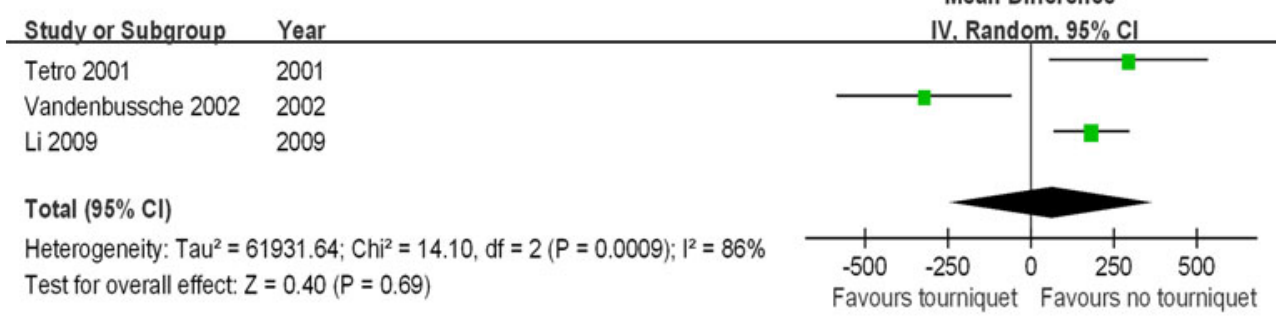

c Intraoperative Blood Loss

\begin{tabular}{llll} 
Study or Subgroup & Year & & IV. Random. $95 \% \mathrm{Cl}$ \\
\hline Aglietti 2000 & 2000 & 2001 & \\
Tetro 2001 & 2002 & 2007 & \\
Kato 2002 & 2009 & & \\
Fukuda 2007 & Li 2009 & & Favours tourniquet Favours no tourniquet
\end{tabular}

d Postoperative Blood Loss

\begin{tabular}{lll|l} 
Study or Subgroup & Year & IV. Random. $95 \% \mathrm{Cl}$ \\
\hline Aglietti 2000 & 2000 & 2001 & \\
Tetro 2001 & 2002 & 2002 & \\
Vandenbussche 2002 & & \\
Wauke 2002 & 2007 & Favours tourniquet Favours no tourniquet \\
Fukuda 2007 & &
\end{tabular}

patients. In the non-tourniquet group, various incidences of embolism were reported during different steps of the operation.

Three studies [1, 33, 36] reported wound problems, including significant hematoma, wound oozing, skin blistering, bruising, superficial wound infection requiring antibiotics treatment, and skin necrosis. The tourniquet group was found to have a higher incidence of postoperative wound problems than the non-tourniquet group (risk ratio: $3.04, P=0.008,95 \% \mathrm{CI}: 1.33-6.95)$.
Eight studies reported a comparison of operation times between the two groups [2, 5, 12, 18, 23, 33, 35, 37]. Subgroup analysis was done according to the timing of releasing tourniquet. Using the tourniquet with early release (deflation after cementing for hemostasis) did not shorten the operation time. However, late release of tourniquet (deflation after wound closure and dressing) would shorten the operation time compared with non-tourniquet groups (Fig. 3). The overall pooling results also found significant shorter operation time in tourniquet group. 
Fig. 2 Results of the metaanalysis of overall clinical thromboembolic events

(a) showed the risk of overall thromboembolic complications was increased by using the tourniquet, although there was no significant difference in the subgroup analysis of clinical pulmonary embolism (b) and clinical deep vein thrombosis (c). The two studies of sonographic deep vein thrombosis (d) showed different results of incidence. Fukuda et al. showed a high incidence in both groups $(77.8 \%(21 / 27)$ in the tourniquet group and $85.7 \%$ $(18 / 21)$ in the non-tourniquet group, respectively), whereas the other study reported a low incidence (1/37 in the tourniquet group and $0 / 40$ in the nontourniquet group, respectively) a Thromboembolic Events

Risk Ratio

\begin{tabular}{ll} 
Study or Subgroup & Year \\
\hline Abdel-Salam 1995 & 1995 \\
Harvey 1997 & 1997 \\
Kato 2002 & 2002 \\
Wauke 2002 & 2002 \\
Vandenbussche 2002 & 2002 \\
Nishiguchi 2005 & 2005 \\
Fukuda 2007 & 2007
\end{tabular}

Total $(95 \% \mathrm{Cl})$

Total events

Heterogeneity: $\mathrm{Chi}^{2}=3.81, \mathrm{df}=6(\mathrm{P}=0.70) ; \mathrm{I}^{2}=0 \%$

Test for overall effect: $Z=2.11(P=0.04)$

b Pulmonary Embolism

\begin{tabular}{ll} 
Study or Subgroup & Year \\
\hline Wauke 2002 & 2002 \\
Kato 2002 & 2002 \\
Nishiguchi 2005 & 2005 \\
Fukuda 2007 & 2007
\end{tabular}

Total $(95 \% \mathrm{Cl})$

Total events

Heterogeneity: $\mathrm{Chi}^{2}=0.99, \mathrm{df}=3(\mathrm{P}=0.80) ; \mathrm{I}^{2}=0 \%$

Test for overall effect: $Z=1.55(P=0.12)$

M-H. Fixed. $95 \% \mathrm{Cl}$

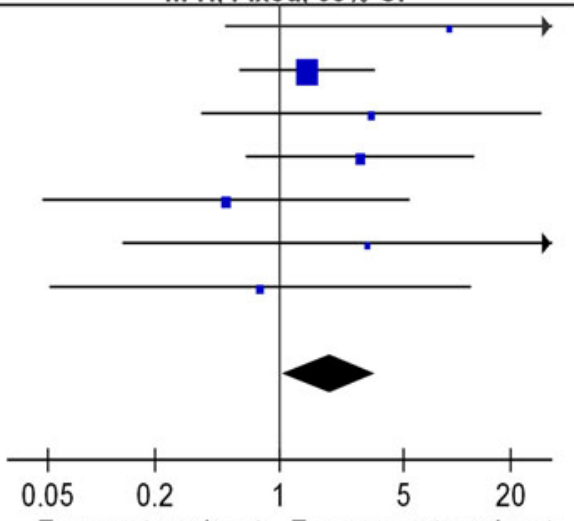

Favours tourniquet Favours no tourniquet

Risk Ratio

M-H. Fixed, $95 \% \mathrm{Cl}$

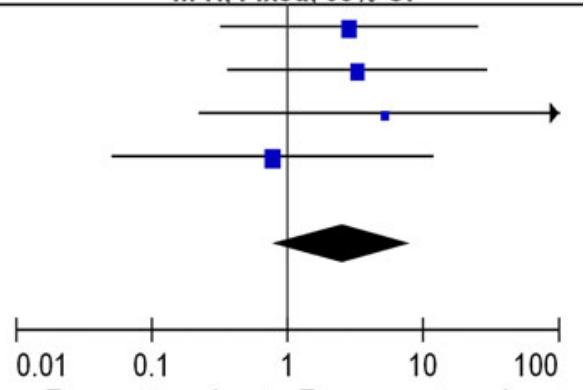

Favours tourniquet Favours no tourniquet

c Clinical DVT

Risk Ratio

Study or Subgroup Year

Abdel-Salam $1995 \quad 1995$

Harvey 1997

Vandenbussche $2002 \quad 2002$

Wauke 2002

Total $(95 \% \mathrm{Cl})$

Total events

Heterogeneity: $\mathrm{Chi}^{2}=2.73, \mathrm{df}=3(\mathrm{P}=0.44) ; \mathrm{I}^{2}=0 \%$

Test for overall effect: $Z=1.56(P=0.12)$

M-H. Fixed. $95 \% \mathrm{Cl}$

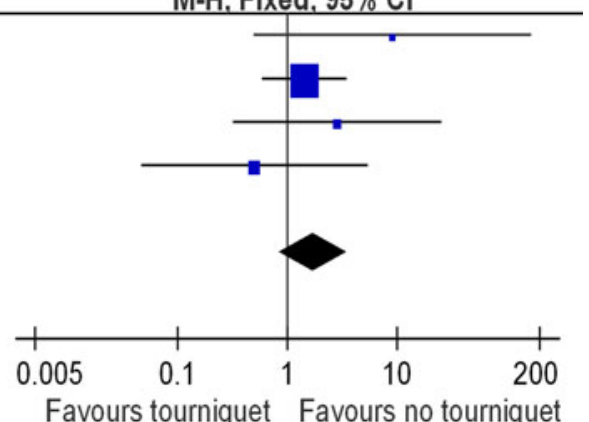

d Sonographic DVT

\begin{tabular}{ll} 
Study or Subgroup & Year \\
\hline Wakankar 1999 & 1999 \\
Fukuda 2007 & 2007
\end{tabular}

Total $(95 \% \mathrm{Cl})$

Total events

Heterogeneity: $\mathrm{Chi}^{2}=0.92, \mathrm{df}=1(\mathrm{P}=0.34) ; \mathrm{I}^{2}=0 \%$

Test for overall effect: $Z=0.26(P=0.79)$
Favours tourniquet Favours no tourniquet

\section{Odds Ratio}

M-H. Fixed, 95\% Cl

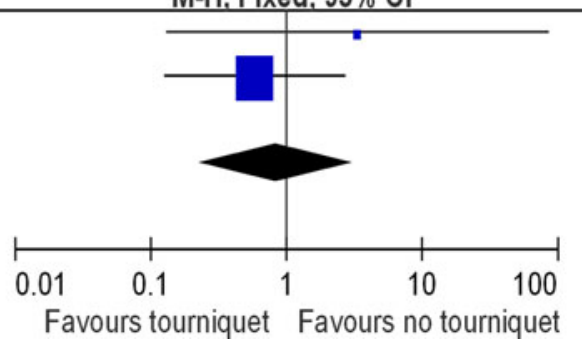


Table 3 Risk ratio of thromboembolic events

\begin{tabular}{llllll}
\hline & \multicolumn{2}{l}{ Incidence } & \multirow{2}{*}{ Risk ratio (95\% CI) } & $\begin{array}{l}\text { Number needed to harm } \\
\text { (NNH) for using a tourniquet }\end{array}$ \\
\cline { 2 - 3 } & Using a tourniquet & No tourniquet & & \\
\hline Clinical thromboembolic events & $13.0 \%(27 / 208)$ & $6.1 \%(12 / 196)$ & $1.91(1.05-3.49)$ & 14.5 \\
Symptomatic deep vein thrombosis & $14.0 \%(19 / 135)$ & $7.1 \%(9 / 126)$ & $1.76(0.87-3.56)$ & 14.5 & 15.6 \\
Symptomatic pulmonary embolism & $9.8 \%(8 / 82)$ & $3.4 \%(3 / 88)$ & $2.54(0.78-8.25)$ & 22.7 \\
Sonographic deep vein thrombosis & $34.4 \%(22 / 64)$ & $30.0 \%(18 / 61)$ & $0.84(0.23-3.09)$ & \\
\hline
\end{tabular}

The risk ratios were calculated by using the fixed effect Mantel-Haensezl method. Each study had a different weight Clinical thromboembolic events included symptomatic deep vein thrombosis and symptomatic pulmonary embolism

Fig. 3 Results of meta-analysis of operation time and the following subgroup analysis. Three studies with early release of tourniquet showed no difference between tourniquet and non-tourniquet groups. Five studies with late release of tourniquet showed shorter operation time in tourniquet group

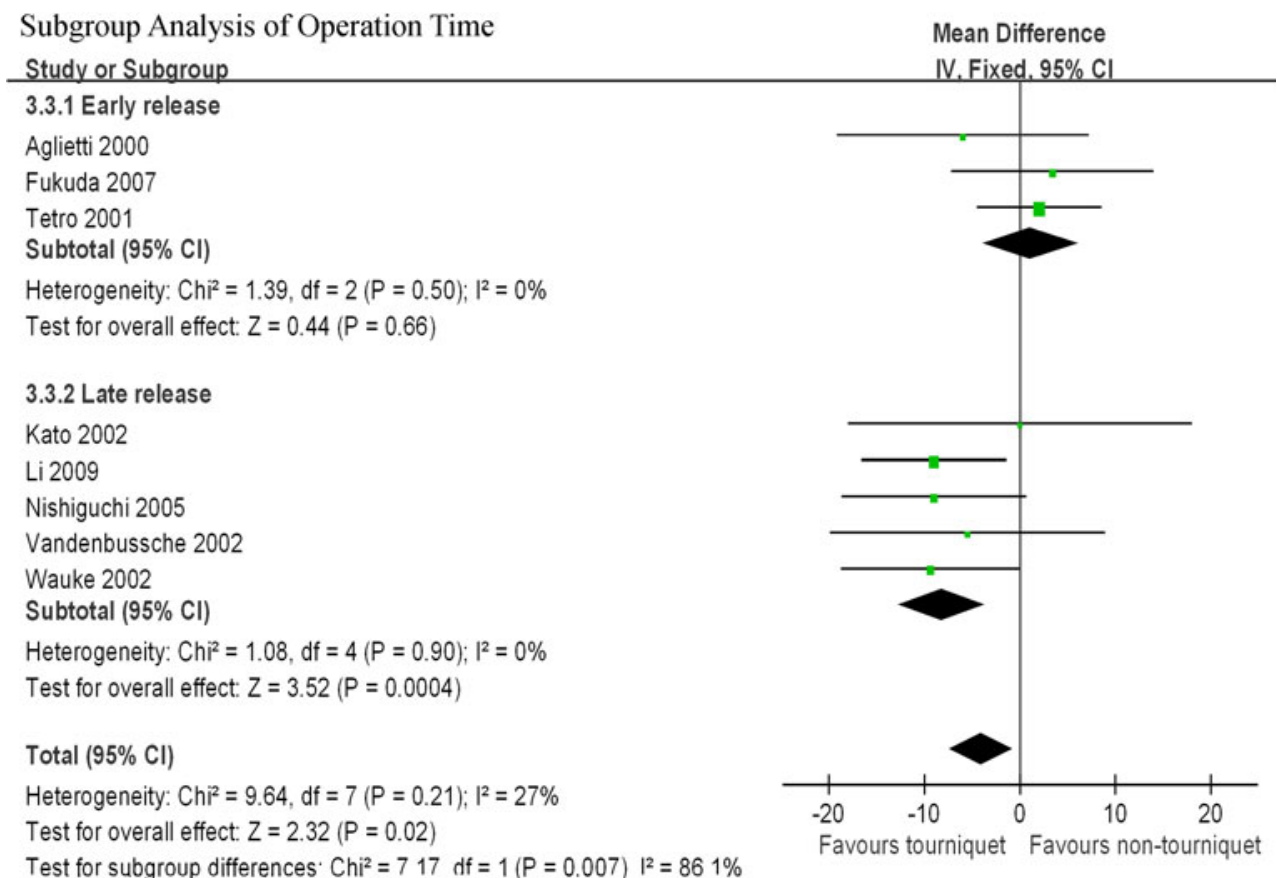

\section{Discussion}

The most important finding of the present meta-analysis was that the true blood loss in TKA was not reduced using a tourniquet, although intraoperative blood loss was significantly less in tourniquet group. In addition, more thromboembolic events and wound complications were identified in the tourniquet group. The effectiveness and safety profile of the tourniquet are questionable.

The pooling data showed that the patients with whom a tourniquet was used in TKA had less total measured operative blood loss. However, we now know that the total measured blood loss represents only a fraction of the true blood loss. The new concept of "calculated blood loss" was introduced using Gross' method [6]. Various studies have indicated that this method likely provides a much more accurate estimation of the true blood loss, than measured blood loss $[27,30]$. The pooling results in the meta-analysis found no significant difference between the two groups. Two current studies even showed that using a tourniquet caused more calculated blood loss [18, 33]. Therefore, we concluded that using a tourniquet does not reduce the overall blood loss effectively.

The results of this analysis also confirmed that there is often a substantial hidden or unmeasured blood loss in TKA [28]. The intraoperative and total measured blood loss were significantly less in the tourniquet group than in the non-tourniquet group, but the pooling results of calculated blood loss showed no significant difference. This phenomenon suggests that TKA involves some blood loss that is not recognized in the routine assessment of either intraoperative blood loss or the postoperative drainage amount, especially when using a tourniquet during the operation [18]. Surgeons tended to underestimate the surgical blood loss in this situation. In fact, reactive blood flow increases with the peak flow appearing within $5 \mathrm{~min}$ 
after the tourniquet is released [16]. This finding supports the notion that patients with whom a tourniquet is used might have more hidden blood loss after the operation.

Thromboembolism is one of the most common complications after TKA and is of great concern because of the associated increases in morbidity and mortality reported in the literature. In the meta-analysis, we found the incidence of clinical thromboembolic events was higher in patients managed with a tourniquet than in those without it. The critical point is the deflation of the tourniquet right after the operation. Transesophageal echocardiography demonstrated shower-like echogenic materials circulating from the lower limbs to the right atrium, ventricle, and pulmonary artery after release of the tourniquet $[12,37]$. This phenomenon lasted for several minutes. The histological examination of these materials showed only thrombi, and no fat, bone marrow, or bone cement [3]. Fortunately, the sonographic phenomenon of embolism was not always clinically relevant. Although the transesophageal echocardiography was thought to be over sensitive, these results hinted us that the thromboembolism was an important issue requiring careful management. To date, no approach to venous thromboembolic prophylaxis has been universally accepted by orthopedic surgeons. The methods of prophylaxis varied among the included studies. Our results suggested that this issue should be of great concern in TKA performed with tourniquet inflation.

The circulatory stasis caused by tourniquet inflation worsened the local soft tissue condition and might have impaired wound healing. Fortunately, most of the complications were mild. Other confounding factors also influenced the soft tissue condition and wound healing. Further studies are needed to investigate the effect of the tourniquet on wound healing.

Regarding the operation time, we should take the timing of tourniquet deflation into account. The tourniquet was released after wound closure and compressive dressing in five studies [12, 18, 23, 35, 37]. The pooling result of the operation time among them showed significantly shorter in the tourniquet group. Three studies with early release of tourniquet did not report this benefit $[2,5,33]$. This finding was consistent with our experience and the recent trials [34]. Releasing the tourniquet right after cementing the prosthesis for hemostasis would cause longer operation time than releasing it after wound closure. However, the influence of the timing of release on blood loss is still controversial $[25,29]$.

The bias of these studies might come from the measurement of intraoperative blood loss. Most of surgeon counted intraoperative blood loss according to the amount of blood in suction bags. This method might underestimate the blood loss when the tourniquet was not used. Several included articles mentioned how they accurately measured the intraoperative blood loss $[2,7,18,33]$. Most of them counted the suction amount and weighed sponges or gauzes in the operative room. The other perioperative management may also impact on blood loss. Some examples are number of drains, timing of removal of drains, temporary clamping of drains or not, and type of postoperative compressive dressing (Table 2). No author temporarily clamped the drain after the operations in our included studies. We did not found the methods of drainage and dressing as confounding factors in our results.

We evaluated the quality of the included studies using the CONSORT statement, which included 22 items for the analysis of randomized controlled trials. The lack of any of the items is associated with biased estimates of the treatment effect, because the information is essential to judge the reliability or relevance of the findings [22]. Our assessment revealed that the weakness of the included randomized studies was mainly the poor methods or description of randomization. Two of the three prospective non-randomized studies allocated the consecutive patients into tourniquet or non-tourniquet groups based on the date of operation to minimize the bias [5, 23]. The other study was part of a double-blind thromboprophylaxis trial [7]; no clear statement of criteria for patient selection was reported.

The major limitation of this analysis is the small sample size. We could not perform statistical tests to eliminate the publication bias. However, such bias was minimized by our comprehensive search, duplicate selection process, and evaluation of methodology. One article reported the operation time as mean and ranges rather than standard deviations [35]. We treated the results as normally distributed data, so the standard deviations were estimated to be onequarter of the ranges of the data values [9]. This estimation might generate bias. However, excluding this study did not change the conclusion of the pooled results.

Although some reports opposing the use of the tourniquet in TKA have been published, the issue remains controversial and the technique is still widely used by surgeons when performing TKA. We suggested that the tourniquet should not be used in patients with high risks of thromboembolism. The thromboprophylaxis and closely monitoring the patients postoperatively are mandatory if the tourniquet was using in TKA. In addition, the hidden blood loss should be kept in mind due to the reactive blood flow after deflation of the tourniquet. The postoperative hemoglobin and hematocrit levels should always be checked to evaluate the blood loss and the need for blood transfusion.

\section{Conclusion}

This meta-analysis provided the best evidence regarding the application of the tourniquet in TKA to date because 
the sample size was far more than those in each single study. The available evidence indicated that although using the tourniquet provided good visualization of the surgical field, it could not reduce the actual blood loss. It saved the operation time only when it was released after wound closure and dressing. The tourniquet might be associated with an increased incidence of thromboembolic events and wound complications. When using the tourniquet in TKA, the surgeons should carefully consider its effectiveness and safety. Inflating the tourniquet only while cementing or for limited time might be an option. Additional well-designed randomized controlled trials are required to clarify the roles of tourniquet and compare the effects of different methods of tourniquet applications in TKA.

Open Access This article is distributed under the terms of the Creative Commons Attribution Noncommercial License which permits any noncommercial use, distribution, and reproduction in any medium, provided the original author(s) and source are credited.

\section{References}

1. Abdel-Salam A, Eyres KS (1995) Effects of tourniquet during total knee arthroplasty. A prospective randomised study. J Bone Joint Surg Br 77:250-253

2. Aglietti P, Baldini A, Vena LM, Abbate R, Fedi S, Falciani M (2000) Effect of tourniquet use on activation of coagulation in total knee replacement. Clin Orthop Relat Res 371:169-177

3. Berman AT, Parmet JL, Harding SP, Israelite CL, Chandrasekaran K, Horrow JC, Singer R, Rosenberg H (1998) Emboli observed with use of transesophageal echocardiography immediately after tourniquet release during total knee arthroplasty with cement. J Bone Joint Surg Am 80:389-396

4. DerSimonian R, Laird N (1986) Meta-analysis in clinical trials. Control Clin Trials 7:177-188

5. Fukuda A, Hasegawa M, Kato K, Shi D, Sudo A, Uchida A (2007) Effect of tourniquet application on deep vein thrombosis after total knee arthroplasty. Arch Orthop Trauma Surg 127:671-675

6. Gross JB (1983) Estimating allowable blood loss: corrected for dilution. Anesthesiology 58:277-280

7. Harvey EJ, Leclerc J, Brooks CE, Burke DL (1997) Effect of tourniquet use on blood loss and incidence of deep vein thrombosis in total knee arthroplasty. J Arthroplasty 12:291-296

8. Higgins JP, Thompson SG, Deeks JJ, Altman DG (2003) Measuring inconsistency in meta-analyses. BMJ 327:557-560

9. Higgins JPT, Green S (2008) Cochrane handbook for systematic reviews of interventions version 5.0.2. The Cochrane Collaboration. Available from www.cochrane-handbook.org. Accessed 1 Dec 2009

10. Horlocker TT, Hebl JR, Gali B, Jankowski CJ, Burkle CM, Berry DJ, Zepeda FA, Stevens SR, Schroeder DR (2006) Anesthetic, patient, and surgical risk factors for neurologic complications after prolonged total tourniquet time during total knee arthroplasty. Anesth Analg 102:950-955

11. Husted H, Toftgaard Jensen $T$ (2005) Influence of the pneumatic tourniquet on patella tracking in total knee arthroplasty: a prospective randomized study in 100 patients. J Arthroplasty 20:694-697

12. Kato N, Nakanishi K, Yoshino S, Ogawa R (2002) Abnormal echogenic findings detected by transesophageal echocardiography and cardiorespiratory impairment during total knee arthroplasty with tourniquet. Anesthesiology 97:1123-1128

13. Katsumata S, Nagashima M, Kato K, Tachihara A, Wauke K, Saito S, Jin E, Kawanami O, Ogawa R, Yoshino S (2005) Changes in coagulation-fibrinolysis marker and neutrophil elastase following the use of tourniquet during total knee arthroplasty and the influence of neutrophil elastase on thromboembolism. Acta Anaesthesiol Scand 49:510-516

14. Komatsu T, Ishibashi Y, Otsuka H, Nagao A, Toh S (2003) The effect of surgical approaches and tourniquet application on patellofemoral tracking in total knee arthroplasty. J Arthroplasty $18: 308-312$

15. Kumar SN, Chapman JA, Rawlins I (1998) Vascular injuries in total knee arthroplasty. A review of the problem with special reference to the possible effects of the tourniquet. J Arthroplasty $13: 211-216$

16. Larsson J, Lewis DH, Liljedahl SO, Lofstrom JB (1977) Early biochemical and hemodynamic changes after operation in a bloodless field. Eur Surg Res 9:311-320

17. Lau J, Ioannidis JP, Schmid CH (1997) Quantitative synthesis in systematic reviews. Ann Intern Med 127:820-826

18. Li B, Wen Y, Wu H, Qian Q, Lin X, Zhao H (2009) The effect of tourniquet use on hidden blood loss in total knee arthroplasty. Int Orthop 33:1263-1268

19. Lombardi AV Jr, Berend KR, Mallory TH, Dodds KL, Adams JB (2003) The relationship of lateral release and tourniquet deflation in total knee arthroplasty. J Knee Surg 16:209-214

20. Lotke PA, Faralli VJ, Orenstein EM, Ecker ML (1991) Blood loss after total knee replacement. Effects of tourniquet release and continuous passive motion. J Bone Joint Surg Am 73:1037-1040

21. Moher D, Cook DJ, Eastwood S, Olkin I, Rennie D, Stroup DF (1999) Improving the quality of reports of meta-analyses of randomised controlled trials: the quorom statement. Quality of reporting of meta-analyses. Lancet 354:1896-1900

22. Moher D, Schulz KF, Altman DG (2001) The consort statement: revised recommendations for improving the quality of reports of parallel-group randomised trials. Lancet 357:1191-1194

23. Nishiguchi M, Takamura N, Abe Y, Kono M, Shindo H, Aoyagi K (2005) Pilot study on the use of tourniquet: a risk factor for pulmonary thromboembolism after total knee arthroplasty? Thromb Res 115:271-276

24. Palmer SH, Graham G (1994) Tourniquet-induced rhabdomyolysis after total knee replacement. Ann R Coll Surg Engl 76:416-417

25. Rama KR, Apsingi S, Poovali S, Jetti A (2007) Timing of tourniquet release in knee arthroplasty. Meta-analysis of randomized, controlled trials. J Bone Joint Surg Am 89:699-705

26. Reikeras O, Clementsen T (2009) Time course of thrombosis and fibrinolysis in total knee arthroplasty with tourniquet application. Local versus systemic activations. J Thromb Thrombolysis 28:425-428

27. Sasanuma H, Sekiya H, Takatoku K, Takada H, Sugimoto N, Hoshino Y (2010) Efficient strategy for controlling postoperative hemorrhage in total knee arthroplasty. Knee Surg Sports Traumatol Arthrosc. doi:10.1007/s00167-010-1263-5

28. Sehat KR, Evans RL, Newman JH (2004) Hidden blood loss following hip and knee arthroplasty. Correct management of blood loss should take hidden loss into account. J Bone Joint Surg Br 86:561-565

29. Steffin B, Green-Riviere E, Giori NJ (2009) Timing of tourniquet release in total knee arthroplasty when using a postoperative blood salvage drain. J Arthroplasty 24:539-542

30. Stucinskas J, Tarasevicius S, Cebatorius A, Robertsson O, Smailys A, Wingstrand H (2009) Conventional drainage versus four hour clamping drainage after total knee arthroplasty in 
severe osteoarthritis: a prospective, randomised trial. Int Orthop 33:1275-1278

31. Stulberg BN, Insall JN, Williams GW, Ghelman B (1984) Deepvein thrombosis following total knee replacement. An analysis of six hundred and thirty-eight arthroplasties. J Bone Joint Surg Am 66:194-201

32. Tamvakopoulos GS, Toms AP, Glasgow M (2005) Subcutaneous thigh fat necrosis as a result of tourniquet control during total knee arthroplasty. Ann R Coll Surg Engl 87:W11-W13

33. Tetro AM, Rudan JF (2001) The effects of a pneumatic tourniquet on blood loss in total knee arthroplasty. Can J Surg 44:33-38

34. Thorey F, Stukenborg-Colsman C, Windhagen H, Wirth CJ (2008) The effect of tourniquet release timing on perioperative blood loss in simultaneous bilateral cemented total knee arthroplasty: a prospective randomized study. Technol Health Care $16: 85-92$

35. Vandenbussche E, Duranthon LD, Couturier M, Pidhorz L, Augereau B (2002) The effect of tourniquet use in total knee arthroplasty. Int Orthop 26:306-309

36. Wakankar HM, Nicholl JE, Koka R, D'Arcy JC (1999) The tourniquet in total knee arthroplasty. A prospective, randomised study. J Bone Joint Surg Br 81:30-33

37. Wauke K, Nagashima M, Kato N, Ogawa R, Yoshino S (2002) Comparative study between thromboembolism and total knee arthroplasty with or without tourniquet in rheumatoid arthritis patients. Arch Orthop Trauma Surg 122:442-446 